\title{
The role of microglia in adult hippocampal neurogenesis
}

\author{
Carmelina Gemma ${ }^{*}$ and Adam D. Bachstetter ${ }^{2}$ \\ Department of Anesthesiology and Pain Medicine, University of Washington, Seattle, WA, USA \\ ${ }^{2}$ Sanders-Brown Center on Aging, University of Kentucky, Lexington, KY, USA
}

Edited by:

Amanda Sierra, University of the Basque Country UPV/EHU, Spain

\section{Reviewed by:}

Juan M. Encinas, University of the

Basque Country UPV/EHU, Spain

Eric D. Laywell, The Florida State

University College of Medicine, USA

\section{*Correspondence:}

Carmelina Gemma, Department of

Anesthesiology and Pain Medicine,

University of Washington, 908

Jefferson Street, Seattle, WA 98001 ,

USA

e-mail: cgemma@u.washington.edu
Our view of microglia has dramatically changed in the last decade. From cells being "silent" in the healthy brain, microglia have emerged to be actively involved in several brain physiological functions including adult hippocampal neurogenesis, and cognitive and behavioral function. In light of recent discoveries revealing a role of microglia as important effectors of neuronal circuit reorganization, considerable attention has been focused on how microglia and hippocampal neurogenesis could be an interdependent phenomenon. In this review the role of microglia in the adult hippocampal neurogenesis under physiological condition is discussed.

Keywords: microglia, CX3CR1, neurogenesis, fractalkine, chemokines

\section{ADULT NEUROGENESIS: THE GENERATION OF NEW NEURONS IN THE MATURE CNS}

Neurogenesis occurs throughout life in the adult mammalian brain including humans (Eriksson et al., 1998; Roy et al., 2000; Wang et al., 2011). In rodents, neurogenesis occurs predominantly in the subgranular zone (SGZ) of the dentate gyrus in the hippocampal formation, and in the subventricular zone (SVZ) of the lateral ventricle (Gage, 2000). The process of generating new neurons consists of four phases: proliferation, migration, differentiation, and survival. Studies over the last decade have elegantly described each step in the neurogenic process (for review see Ming and Song, 2011). Adult hippocampal neurogenesis originates from a population of proliferating radial and nonradial precursor cells located in the SGZ, which give rise to neural progenitor cells (NPCs), which in turn generate neuroblasts (Ehninger and Kempermann, 2008). Immature neurons migrate into the granule cell layer and differentiate into dentate granule cell in the hippocampus. Although NPCs proliferation generates a vast number of neurons only a very small proportion survives for long period of time (Kempermann et al., 2003). Indeed, most of the newly born cells are eliminated by apoptosis during first few days following birth. The cells that do survive for the first two weeks are then stable and integrated into the network of the dentate gyrus throughout life. After this time point, only very small changes in cell number occur. There are only two critical periods for neural progeny survival: (1) during transition from amplifying neuroprogenitors to neuroblast (Platel et al., 2010; Sierra et al., 2010); and (2) during the integration stage of the immature neurons (Tashiro et al., 2006; Mouret et al., 2008). By two months the surviving neurons receive input from other neurons (van et al., 2002; Piatti et al., 2006), with some forming functional synapses, and possessing electrophysiological properties indistinguishable from those of mature neurons (Ge et al., 2008).

\section{MICROGLIA HETEROGENEITY}

Microglia are recognized as the resident brain immune cells and have important roles in the healthy central nervous system (CNS). For example as the brain's tissue macrophage, a primary function of microglia is to phagocytose dying cells and cellular debris silently (i.e., without producing inflammation). However, upon a pathologic insult, such as infection or brain injury, microglia respond rapidly. Part of this reactive response includes a morphological change in appearance. In the healthy CNS, microglia have highly ramified morphology with thin processes, which dynamically move in the brain parenchyma in what has been called a surveillance state (Nimmerjahn et al., 2005). We define microglia in this surveillance state as ramified microglia. In contrast, reactive microglia, (i.e., microglia that are no longer ramified microglia) can adopt a number of altered morphologies, including a hypertrophic cell with enlarged processes, or amoeboid macrophage like morphology.

\section{MICROGLIA: ROLE OF RAMIFIED MICROGLIA IN ADULT NEUROGENESIS}

Increasing evidence suggests that ramified microglia are an essential component of the neurogenic niche in the SGZ of the adult hippocampus. One of the critical roles of microglia in modulating hippocampal neurogenesis is pruning of newborn cells during the first critical period of survival. Sierra et al. (2010) demonstrated that ramified microglia have an important function in phagocytosis of apoptotic cells during the first days of their life. As the newborn cells are integrated into the existing circuits other cells become apoptotic. The apoptotic cells are subsequently removed by microglia in an immunologically silently process (i.e., without inflammation). In response to tissue damage, such as a traumatic brain injury, damage associated molecular patterns (DAMPs: e.g., Adenosine triphosphate (ATP), DNA) are released and cause microglia to become activated towards a proinflammatory state. 
In the context of neurogenesis, the dying cells undergo a process of programmed cell death, and no DAMPs are released. As opposed to the phagocytosis by ameboid microglia observed during trauma or neurodegeneration, phagocytosis of apoptotic cells during neurogenesis is performed by ramified microglia. Ramified microglia remove apoptotic neurons by a special modification of the microglial process, which form phagocytic pouches that engulf the apoptotic cells. The phagocytic pouches, occurs independent from the cell body, in terminal or en passant branches, as opposed to engulfment of the soma by ameboid microglia. Furthermore, phagocytosis of apoptotic neurons by ramified microglia is highly efficient as demonstrated by the high proportion of apoptotic cells engulfed by ramified microglia, the proportion of microglia engaged in the engulfing process, and the time to completely eliminate apoptotic cells (Sierra et al., 2010).

\section{MICROGLIA PROVIDE TROPHIC SUPPORT FOR ADULT HIPPOCAMPAL NEUROGENESIS}

The role of microglia in neurogenesis is not limited to removal of cells. Evidence suggests that microglia secrete factors that can influence proliferation, differentiation into neurons or glia, and survival of the newborn cell. How could microglia-derived factors influence neurogenesis? Several different mechanisms have been proposed: (1) microglia could have a direct instructive role in dictating the commitment to a neuronal phenotype, (2) microglia could promote proliferation through secretion of neurotrophic factors, and (3) microglia could produce factors that regulate survival of neuronal cells. In favor of a direct instructive role of microglia to direct neuronal differentiation, in vitro studies demonstrate that microglia have the capacity to guide the differentiation of precursor cells isolated from embryonic brain as well as adult mouse neural precursor cells toward a neuronal phenotype (Aarum et al., 2003). In addition, precursor cell cultures grown in conditioned media from microglia cells contain a higher proportion of neurons than what would be expected from their spontaneous differentiation alone. Yet, microglia can affect proliferation and survival, in addition to neuron differentiation. For example, Morgan et al. (2004) further investigated the effect of non-stimulated microglia on neuronal proliferation and survival. The authors used microglia-conditioned medium collected from primary rat microglia to treat neurons in vitro for 7 days and found that the microglia-conditioned medium induced a $50 \%$ increase in neuronal survival compared to untreated neurons. A different study confirmed and extended these findings showing that addition of microglia-conditioned media in SVZ-derived culture increased neuroblast production (Walton et al., 2006). Furthermore, loss of inducible neurogenesis was paralleled by microglia depletion in proliferating culture. While a number of growth factors secreted by microglia could be responsible for such effect, evidence suggests that microglia are capable of producing growth factors, such as Insulin-like growth factor 1 (IGF-1) and Brain-derived neurotrophic factor (BDNF), which promote neurogenesis (Ziv and Schwartz, 2008). Figure 1 highlights the known ways microglia are involved in the neurogenic process.

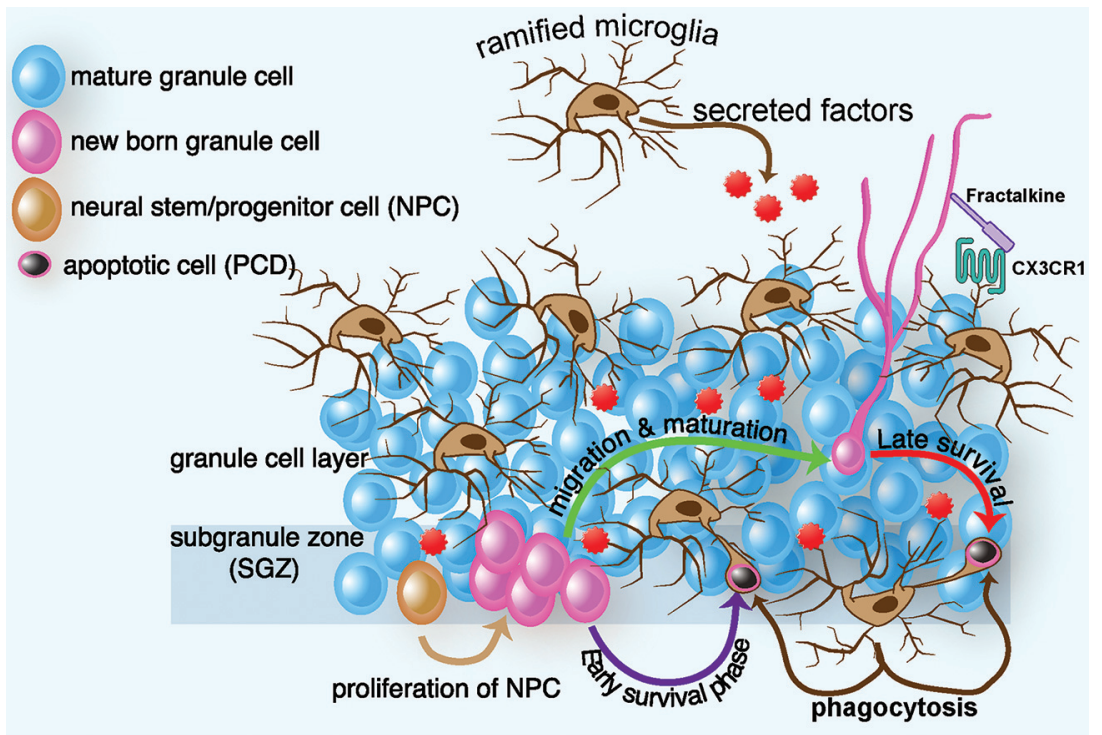

FIGURE 1 | Schematic diagram of ramified microglia and their effect on adult hippocampal neurogenesis. In intact brain, microglia regulate several steps of adult hippocampal neurogenesis. In the SGZ, progenitor cells migrate to the granule cell layer and differentiate into a neuronal phenotype, with most NPCs dying in the first few days of life. Within two months, the surviving neurons receive input, form functional synapses with their target cells, and exhibit electrophysiological properties indistinguishable from those of mature neurons. In intact brain, ramified microglia eliminate apoptotic newborn cells during the first few days of their life by phagocytosis. This phagocytosis occurs by a special modification of the microglial processes which form phagocytic pouches that engulf the apoptotic cells. Microglia can also affect proliferation, differentiation, and survival, through the secretion of neurotrophic factors. Finally microglia communicate with nearby neurons through the CX3CR1/CX3CL1 signaling. Interactions between CX3CL1 and CX3CR1 contribute to the ability of microglia to maintain a surveillant/ramified phenotype. Disruption of this signaling results in a change in microglia phenotype and function, which leads to decreased hippocampal neurogenesis. 
In rodents, environmental enrichment has been one of the most clear and reproducible ways to stimulate adult neurogenesis (van et al., 1999; Inokuchi, 2011). Environmental enrichment has many beneficial effects on CNS function; intriguingly, some of the effects of environmental enrichment may be mediated by microglia. A recent finding showed that when microglia extracted from the hippocampus of runner mice were added to the hippocampal preparation of sedentary mice, the number of NPCs in the hippocampal culture of sedentary mice increased (Vukovic et al., 2012), suggesting that microglia are intrinsically altered or "primed" by the enriching experience. Indeed, following an enriched environment or physical activity, beneficial microglia increase, and this increase correlates with an increase in hippocampal neurogenesis (Ziv et al., 2006; Choi et al., 2008). However, other studies have shown no correlation or an inverse correlation in the role of microglia in neurogenesis stimulated by environmental enrichment (Gebara et al., 2013).

\section{NEURON-MICROGLIA DIALOGUE IN ADULT HIPPOCAMPAL NEUROGENESIS}

Until recently, neurons were believed to be submissive to the effects of microglia. However, a number of neuronal signals were found that can regulate microglia activation (Biber et al., 2007), suggesting a neuron-microglia dialogue. Indeed, neurons may also deliver signals that keep microglia in their surveillance mode indicating normal function. Under physiological conditions several neuron-mediated signals have an anti-inflammatory action at the level of the microglia. Cluster of differentiation (CD) 200 (also called OX2), CD47, CD55, CX3CL1 (fractalkine), are all neuroimmunoregulatory proteins constitutively expressed in healthy neurons with a cognate receptor on microglia (Kierdorf and Prinz, 2013). In the context of adult hippocampal neurogenesis, the question to be considered is whether neurons in the neurogenic niche communicate with microglia to regulate neurogenesis. A recent report suggests that the NPCs could secrete factors that regulate microglia function. Using an in vitro assay, the conditioned medium from NPCs caused microglia to increase in numbers, migrate to the site of injury, and to become phagocytic (Mosher et al., 2012). As previously mentioned, microglia can regulate neurogenesis at a number of steps in the neurogenic process. Therefore, a bidirectional regulation of neurons/neurogenesis and microglia might provide a means to fine tune the neurogenic process.

\section{FRACTALKINE/CX3CR1 AS AN EXAMPLE OF NEURON-MICROGLIA DIALOGUE}

As noted, ramified microglia dynamically move their processes within a volume of parenchyma and, in normal conditions, target synaptic structure (Wake et al., 2009; Tremblay et al., 2010). How do microglia know where to move their processes? What signals do microglia receive when they survey the neurons? Are there different signals for synaptic pruning or neuron pruning? Some of these questions are beginning to be answered, but this is an exciting area of microglia biology that is largely unexplored.

One of the best-characterized examples of a neuronal signal that regulates microglia function is the chemokine fractalkine. The anatomical expression of fractalkine on neurons and CXRCR1 on microglia led to the hypothesis of a unique signaling whereby neurons may maintain microglia in a surveillant/ramified state through a repressive fractalkine signal. Fractalkine is constitutively expressed at high levels on healthy neurons. The receptor for fractalkine, CX3CR1, is more highly expressed on microglia, than macrophages. Over the past decade numerous investigators have provided strong support that fractalkine does suppress microglia activation (Cardona et al., 2006; Ransohoff et al., 2007; Bhaskar et al., 2010; Lee et al., 2010).

Interestingly, data suggest that fractalkine signaling may be involved in neuron-microglia dialogue in the neurogenic niche that regulates neurogenesis. First it was shown by genetic deletion or pharmacological antagonism of CX3CR1 in young adult rats that CX3CR1 was important for the maintenance of hippocampal neurogenesis, as animals with decreased CX3CR1 have less neurogenesis (Bachstetter et al., 2011). Furthermore, levels of fractalkine, which are abundantly expressed in young healthy rodent brains, were decreased in aged rodents (Bachstetter et al., 2011). It was suggested that the decrease in fractalkine signaling may contribute to the increased neuroinflammation and decreased hippocampal neurogenesis seen in the aged rodent brain. To test this hypothesis, aged rats were administered fractalkine, which reversed the age-related decrease in hippocampal neurogenesis and restored microglia to a ramified morphology (Bachstetter et al., 2011).

Loss of fractalkine/CX3CR1 signaling in a non-disease model, not only affects neurogenesis, it can cause impairments in motor learning, cognitive function, and synaptic plasticity through increased microglia activation and inflammation in the CNS (Rogers et al., 2011). Impairment of long-term potentiation (LTP) and neurogenesis likely represent the mechanism responsible for the defect observed in hippocampal-dependent associative and spatial memory formation. However, multiple mechanisms could account for the impairment in cognitive function and synaptic plasticity observed in the CX3CR1-deficient mice. Studies on CX3CR1/fractalkine signaling and neuronal activity have produced discordant results. For example, exogenous application of fractalkine inhibits LTP and LTP impairment failed to occur in CX3CR1 deficient mice (Maggi et al., 2009). Differences in experimental protocols, animal age and gender, and housing conditions etc. could explain the discordance between studies.

As noted earlier, environmental enrichment, including exercise can stimulate neurogenesis. Recent data suggest that fractalkine/CX3CR1 signaling may be involved in the exerciseinduced increase in hippocampal neurogenesis. Exercise reversed the age-related decline in fractalkine hippocampal levels and increased hippocampal neurogenesis (Vukovic et al., 2012). Furthermore, pharmacological antagonism of CX3CR1 prevented the increase in hippocampal neurogenesis (Vukovic et al., 2012). Therefore, the aforementioned results suggest that fractalkine signaling is a way microglia function could be tuned by neurons. A previous interesting study (Maggi et al., 2011) showed that absence of CX3CR1 in female mice leads to an increase in hippocampal plasticity and learning performance and a decrease in hippocampal-dependent response to environmental stimulation. In addition, deficiency of CX3CR1 in female mice blunted the 
positive effect of an enriched environment on neuronal plasticity. These controversial results are quite intriguing as they clearly show a gender difference effect of microglia in synaptic plasticity. Interesting, profound sex differences in the microglia colonization of the developing rodent brain were observed recently. At P4, male rats have significantly more microglia than females in many brain regions critical for cognition, learning, and memory including the hippocampus, parietal cortex, and amygdala (Schwarz and Bilbo, 2012). It would be interesting to know if a gender difference in microglia density exists during adulthood. Taken together these studies demonstrate that microglia through the CX3CR1 receptor play a physiological role in adult hippocampal neurogenesis and cognitive function.

\section{CONCLUSION}

Microglia are an essential component of the neurogenic niche and emerging evidence shows new and fundamental roles for microglia in the control of neuronal proliferation, differentiation, and survival of newborn neurons into the existing neuronal circuitry. However the specific molecular and cellular mechanisms through which microglia regulate different stages of neurogenesis are only beginning to be explored. A deeper understanding of the physiological function of microglia in the different steps of adult neurogenesis is needed.

\section{ACKNOWLEDGMENTS}

This work was supported by the National Institute of Aging (5R21AG039739-03). We thank Paula Thomason for manuscript preparation and editing.

\section{REFERENCES}

Aarum, J., Sandberg, K., Haeberlein, S. L., and Persson, M. A. (2003). Migration and differentiation of neural precursor cells can be directed by microglia. Proc. Natl. Acad. Sci. U S A 100, 15983-15988. doi: 10.1073/pnas.2237050100

Bachstetter, A. D., Morganti, J. M., Jernberg, J., Schlunk, A., Mitchell, S. H., Brewster, K. W., et al. (2011). Fractalkine and CX(3)CR1 regulate hippocampal neurogenesis in adult and aged rats. Neurobiol. Aging 32, 2030-2044. doi: 10. 1016/j.neurobiolaging.2009.11.022

Bhaskar, K., Konerth, M., Kokiko-Cochran, O. N., Cardona, A., Ransohoff, R. M., and Lamb, B. T. (2010). Regulation of tau pathology by the microglial fractalkine receptor. Neuron 68, 19-31. doi: 10.1016/j.neuron.2010.08.023

Biber, K., Neumann, H., Inoue, K., and Boddeke, H. W. (2007). Neuronal 'On' and 'Off' signals control microglia. Trends Neurosci. 30, 596-602. doi: 10.1016/j.tins. 2007.08.007

Cardona, A. E., Pioro, E. P., Sasse, M. E., Kostenko, V., Cardona, S. M., Dijkstra, I. M., et al. (2006). Control of microglial neurotoxicity by the fractalkine receptor. Nat. Neurosci. 9, 917-924. doi: 10.1038/nn1715

Choi, S. H., Veeraraghavalu, K., Lazarov, O., Marler, S., Ransohoff, R. M., Ramirez, J. M., et al. (2008). Non-cell-autonomous effects of presenilin 1 variants on enrichment-mediated hippocampal progenitor cell proliferation and differentiation. Neuron 59, 568-580. doi: 10.1016/j.neuron.2008.07.033

Ehninger, D., and Kempermann, G. (2008). Neurogenesis in the adult hippocampus. Cell Tissue Res. 331, 243-250. doi: 10.1007/s00441-007-0478-3

Eriksson, P. S., Perfilieva, E., Bjork-Eriksson, T., Alborn, A. M., Nordborg, C., Peterson, D. A., et al. (1998). Neurogenesis in the adult human hippocampus. Nat. Med. 4, 1313-1317. doi: 10.1038/3305

Gage, F. H. (2000). Mammalian neural stem cells. Science 287, 1433-1438. doi: 10. 1126/science. 287.5457 .1433

Ge, S., Sailor, K. A., Ming, G. L., and Song, H. (2008). Synaptic integration and plasticity of new neurons in the adult hippocampus. J. Physiol. 586, 3759-3765. doi: 10.1113/jphysiol.2008.155655

Gebara, E., Sultan, S., Kocher-Braissant, J., and Toni, N. (2013). Adult hippocampal neurogenesis inversely correlates with microglia in conditions of voluntary running and aging. Front. Neurosci. 7:145. doi: 10.3389/fnins.2013. 00145

Inokuchi, K. (2011). Adult neurogenesis and modulation of neural circuit function. Curr. Opin. Neurobiol. 21, 360-364. doi: 10.1016/j.conb.2011.02.006

Kempermann, G., Gast, D., Kronenberg, G., Yamaguchi, M., and Gage, F. H. (2003). Early determination and long-term persistence of adult-generated new neurons in the hippocampus of mice. Development 130, 391-399. doi: 10.1242/dev. 00203

Kierdorf, K., and Prinz, M. (2013). Factors regulating microglia activation. Front. Cell. Neurosci. 7:44. doi: 10.3389/fncel.2013.00044

Lee, S., Varvel, N. H., Konerth, M. E., Xu, G., Cardona, A. E., Ransohoff, R. M., et al. (2010). CX3CR1 deficiency alters microglial activation and reduces betaamyloid deposition in two alzheimer's disease mouse models. Am. J. Pathol. 177, 2549-2562. doi: 10.2353/ajpath.2010.100265

Maggi, L., Scianni, M., Branchi, I., D’Andrea, I., Lauro, C., and Limatola, C. (2011). CX(3)CR1 deficiency alters hippocampal-dependent plasticity phenomena blunting the effects of enriched environment. Front. Cell. Neurosci. 5:22. doi: $10.3389 /$ fncel.2011.00022

Maggi, L., Trettel, F., Scianni, M., Bertollini, C., Eusebi, F., Fredholm, B. B., et al. (2009). LTP impairment by fractalkine/CX3CL1 in mouse hippocampus is mediated through the activity of adenosine receptor type 3 (A3R). J. Neuroimmunol. 215, 36-42. doi: 10.1016/j.jneuroim.2009.07.016

Ming, G. L., and Song, H. (2011). Adult neurogenesis in the mammalian brain: significant answers and significant questions. Neuron 70, 687-702. doi: 10 . 1016/j.neuron.2011.05.001

Morgan, S. C., Taylor, D. L., and Pocock, J. M. (2004). Microglia release activators of neuronal proliferation mediated by activation of mitogen-activated protein kinase, phosphatidylinositol-3-kinase/Akt and delta-Notch signalling cascades. J. Neurochem. 90, 89-101. doi: 10.1111/j.1471-4159.2004.02461.x

Mosher, K. I., Andres, R. H., Fukuhara, T., Bieri, G., Hasegawa-Moriyama, M., He, Y., et al. (2012). Neural progenitor cells regulate microglia functions and activity. Nat. Neurosci. 15, 1485-1487. doi: 10.1038/nn.3233

Mouret, A., Gheusi, G., Gabellec, M. M., de, C. F., Olivo-Marin, J. C., and Lledo, P. M. (2008). Learning and survival of newly generated neurons: when time matters. J. Neurosci. 28, 11511-11516. doi: 10.1523/jneurosci.2954-08.2008

Nimmerjahn, A., Kirchhoff, F., and Helmchen, F. (2005). Resting microglial cells are highly dynamic surveillants of brain parenchyma in vivo. Science 308, 1314 1318. doi: 10.1126/science. 1110647

Piatti, V. C., Esposito, M. S., and Schinder, A. F. (2006). The timing of neuronal development in adult hippocampal neurogenesis. Neuroscientist 12, 463-468. doi: $10.1177 / 1073858406293538$

Platel, J. C., Dave, K. A., Gordon, V., Lacar, B., Rubio, M. E., and Bordey, A. (2010). NMDA receptors activated by subventricular zone astrocytic glutamate are critical for neuroblast survival prior to entering a synaptic network. Neuron 65, 859-872. doi: 10.1016/j.neuron.2010.03.009

Ransohoff, R. M., Liu, L., and Cardona, A. E. (2007). Chemokines and chemokine receptors: multipurpose players in neuroinflammation. Int. Rev. Neurobiol. 82, 187-204. doi: 10.1016/s0074-7742(07)82010-1

Rogers, J. T., Morganti, J. M., Bachstetter, A. D., Hudson, C. E., Peters, M. M., Grimmig, B. A., et al. (2011). CX3CR1 deficiency leads to impairment of hippocampal cognitive function and synaptic plasticity. J. Neurosci. 31, 1624116250. doi: 10.1523/jneurosci.3667-11.2011

Roy, N. S., Wang, S., Jiang, L., Kang, J., Benraiss, A., Harrison-Restelli, C., et al. (2000). In vitro neurogenesis by progenitor cells isolated from the adult human hippocampus. Nat. Med. 6, 271-277. doi: 10.1038/73119

Schwarz, J. M., and Bilbo, S. D. (2012). Sex, glia, and development: interactions in health and disease. Horm. Behav. 62, 243-253. doi: 10.1016/j.yhbeh.2012.02.018

Sierra, A., Encinas, J. M., Deudero, J. J., Chancey, J. H., Enikolopov, G., OverstreetWadiche, L. S., et al. (2010). Microglia shape adult hippocampal neurogenesis through apoptosis-coupled phagocytosis. Cell Stem Cell 7, 483-495. doi: 10. 1016/j.stem.2010.08.014

Tashiro, A., Sandler, V. M., Toni, N., Zhao, C., and Gage, F. H. (2006). NMDAreceptor-mediated, cell-specific integration of new neurons in adult dentate gyrus. Nature 442, 929-933. doi: 10.1038/nature05028

Tremblay, M. E., Lowery, R. L., and Majewska, A. K. (2010). Microglial interactions with synapses are modulated by visual experience. PLoS Biol. 8:e1000527. doi: 10.1371/journal.pbio.1000527

van, P. H., Christie, B. R., Sejnowski, T. J., and Gage, F. H. (1999). Running enhances neurogenesis, learning, and long-term potentiation in mice. 
Proc. Natl. Acad. Sci. U S A 96, 13427-13431. doi: 10.1073/pnas.96.23. 13427

van, P. H., Schinder, A. F., Christie, B. R., Toni, N., Palmer, T. D., and Gage, F. H. (2002). Functional neurogenesis in the adult hippocampus. Nature 415, 1030 1034. doi: 10.1038/4151030a

Vukovic, J., Colditz, M. J., Blackmore, D. G., Ruitenberg, M. J., and Bartlett, P. F. (2012). Microglia modulate hippocampal neural precursor activity in response to exercise and aging. J. Neurosci. 32, 6435-6443. doi: 10.1523/jneurosci.592511.2012

Wake, H., Moorhouse, A. J., Jinno, S., Kohsaka, S., and Nabekura, J. (2009). Resting microglia directly monitor the functional state of synapses in vivo and determine the fate of ischemic terminals. J. Neurosci. 29, 3974-3980. doi: 10.1523/jneurosci. 4363-08.2009

Walton, N. M., Sutter, B. M., Laywell, E. D., Levkoff, L. H., Kearns, S. M., Marshall, G. P., et al. (2006). Microglia instruct subventricular zone neurogenesis. Glia 54, 815-825. doi: 10.1002/glia.20419

Wang, C., Liu, F., Liu, Y. Y., Zhao, C. H., You, Y., Wang, L., et al. (2011). Identification and characterization of neuroblasts in the subventricular zone and rostral migratory stream of the adult human brain. Cell Res. 21, 1534-1550. doi: $10.1038 / \mathrm{cr} .2011 .83$

Ziv, Y., Ron, N., Butovsky, O., Landa, G., Sudai, E., Greenberg, N., et al. (2006). Immune cells contribute to the maintenance of neurogenesis and spatial learning abilities in adulthood. Nat. Neurosci. 9, 268-275. doi: 10.1038/ nn 1629

Ziv, Y., and Schwartz, M. (2008). Immune-based regulation of adult neurogenesis: implications for learning and memory. Brain Behav. Immun. 22, 167-176. doi: 10.1016/j.bbi.2007.08.006

Conflict of Interest Statement: The authors declare that the research was conducted in the absence of any commercial or financial relationships that could be construed as a potential conflict of interest.

Received: 31 January 2013; accepted: 05 November 2013; published online: 22 November 2013.

Citation: Gemma $C$ and Bachstetter AD (2013) The role of microglia in adult hippocampal neurogenesis. Front. Cell. Neurosci. 7:229. doi: 10.3389/fncel.2013.00229 This article was submitted to the journal Frontiers in Cellular Neuroscience.

Copyright (C) 2013 Gemma and Bachstetter. This is an open-access article distributed under the terms of the Creative Commons Attribution License (CC BY). The use, distribution or reproduction in other forums is permitted, provided the original author(s) or licensor are credited and that the original publication in this journal is cited, in accordance with accepted academic practice. No use, distribution or reproduction is permitted which does not comply with these terms. 